\title{
Les Salvadoriennes et la guerre civile révolutionnaire
}

«Grâce à la guerre, nous sommes sorties des cuisines »... Mais pour quoi faire?

Jules FALQUET

\section{CpenEdition}

Journals

\section{Édition électronique}

URL : https://journals.openedition.org/clio/411

DOI : 10.4000/clio.411

ISSN : $1777-5299$

Éditeur

Belin

Édition imprimée

Date de publication : 1 avril 1997

ISBN : 2-85816-323-5

ISSN : $1252-7017$

\section{Référence électronique}

Jules FALQUET, «Les Salvadoriennes et la guerre civile révolutionnaire », Clio. Histoire, femmes et sociétés [En ligne], 5 | 1997, mis en ligne le 01 janvier 2005, consulté le 23 avril 2022. URL : http:// journals.openedition.org/clio/411; DOI : https://doi.org/10.4000/clio.411

Ce document a été généré automatiquement le 23 avril 2022.

Tous droits réservés 


\title{
Les Salvadoriennes et la guerre civile révolutionnaire
}

«Grâce à la guerre, nous sommes sorties des cuisines »... Mais pour quoi faire?

\author{
Jules FALQUET
}

1 Glissant sur les souffrances, la barbarie et la mort, certaines personnes considèrent les guerres comme de formidables accélérateurs de l'histoire qui permettent une modernisation sociale, une redistribution des pouvoirs et - pourquoi pas? - une amélioration du sort des femmes, pour services rendus. Une telle vision est à l'opposée du vécu quotidien de la guerre pour la plupart des personnes qui, placées depuis « toujours » dans une position dominée, n'ont guère de moyens de tirer leur épingle du jeu. Au Salvador, petit pays d'Amérique centrale, douze ans de guerre civile révolutionnaire de 1981 à 1992 entre le Front Farabundo Martí (FMLN, d'obédience marxiste-léniniste) et les gouvernements démocrate-chrétien puis d'extrême droite, puissamment soutenus par les Etats-unis, ont laissé de profondes traces. Pour les femmes, le résultat est extrêmement nuancé. Une des féministes salvadoriennes les plus connues, Candelaria Navas, a publié récemment un recueil de textes intitulé Cela valait la peine?! Nous verrons pourquoi les femmes salvadoriennes ont eu dans cette guerre une participation très importante. Nous évoquerons ensuite le bilan de la guerre pour ces femmes, très différent selon leur situation sociale et leur degré d'engagement. Nous tenterons enfin de donner des pistes sur les rapports complexes entre cette guerre révolutionnaire et l'apparition dans le pays, juste après la guerre, d'un fort mouvement des femmes de plus en plus ouvertement féministe.

2 Participation à la lutte : femmes invisibles, femmes en vue et femmes dans la ligne de mire

3 Si la guérilla du FMLN a pu s'enorgueillir de compter parmi ses quelques douze mille combattant-e-s un tiers de femmes - un pourcentage élevé pour les guérillas latinoaméricaines ${ }^{1}$ - c'est que les femmes étaient, depuis avant la guerre et malgré le poids d'un système patriarcal écrasant, fort actives dans la vie du pays. Les Salvadoriennes sont traditionnellement très nombreuses sur le marché du travail (mal) rémunéré ; 
avant la guerre, elles y étaient obligées par l'abandon des pères de leurs enfants, une pratique massive chez les Salvadoriens. Pendant les années 1960 et 1970, beaucoup ont fait leurs premiers pas dans la participation publique, communautaire, grâce aux activités de la théologie de la libération. Les réunions de lecture critique de la réalité sociale appuyées sur la Bible, les sessions d'alphabétisation, la formation de coopératives leur ont permis, légitimement aux yeux des hommes, de mettre le nez hors du foyer. Depuis 1970, année de la formation de la première organisation de lutte armée révolutionnaire par scission du PC, le «mouvement populaire» croît constamment en force et en radicalité, jusqu'à basculer presque complètement dans le camp de la guérilla, à qui il fournit le gros de ses forces. Or ce mouvement populaire est notablement porté par des femmes. Dans le mouvement paysan-coopérativiste, même si les hommes sont nettement plus visibles, ce sont aussi beaucoup de jeunes filles ou de mères de famille qui, discrètement, portent la parole révolutionnaire, transmettent des messages ou nourrissent la guérilla en formation. Le syndicat enseignant ANDES 21 de junio, extrêmement combattif, prend rapidement la tête du mouvement populaire. Il est composé essentiellement de femmes, souvent d'origine modeste, qui font le lien entre la ville et la campagne. Bien que la direction du syndicat soit largement plus masculine que sa base, la dirigeante principale d'ANDES est une femme : Mélida Anaya Montes. C'est une des premières organisatrices, clandestine, de la lutte armée révolutionnaire, où elle devient la Commandante Ana María, dirigeante en second. Sa détermination, son prestige et sa clairvoyance politique en font, avec le dirigeant ouvrier Caetano Carpio (ancien secrétaire général du parti communiste salvadorien et fondateur de la guérilla sous le nom de Commandant Marcial) un des principaux piliers de la lutte révolutionnaire pendant plus de dix ans. Est-ce par socialisation masculine que Marcial prônait la "guerre populaire prolongée ", sous l'hégémonie du «bloc populaire » et jusqu'à la victoire militaire ? Mélida Anaya Montes, elle, luttait pour une alliance large de tous les secteurs progressistes en vue d'une solution politique au conflit. Toujours est-il qu'en 1983, elle est assassinée sur ordre de Marcial, qui craignait semble-t-il qu'elle ne lui ravisse la direction de la révolution.

4 La participation des femmes à la vie du pays, aux luttes sociales puis à la guerre civile révolutionnaire, a été très importante ${ }^{2}$. Mais il faut garder à l'esprit la différence énorme entre les femmes de classe moyenne urbaine scolarisées, qui commencent alors à avoir accès assez massivement à l'université, et l'immense majorité des paysannes pauvres. Toujours quasi analphabètes, en charge de cinq enfants (en moyenne), beaucoup viennent grossir les rangs de la population marginale urbaine qui cherche quotidiennement sa survie sur les terrains vagues et les gigantesques dépôts d'ordures de la capitale. Un certain nombre de femmes de la ville et d'étudiantes s'engagent dans la lutte armée par idéalisme révolutionnaire, enflammées par un projet de vie nouveau qui inclut des relations de couple et familiales transformées : responsabilité collective des enfants dans les maisons de sécurité, partage plus équitable de certaines tâches. Pour elles, la révolution est aussi, sinon une libération immédiate des femmes, du moins un grand pas vers l'égalité : le parti ne leur confie-t-il pas à elles aussi des armes et des responsabilités politico-militaires ${ }^{3}$ ? Mais pour beaucoup de femmes de la campagne, l'engagement est bien différent. Certaines sont passées insensiblement des causeries sur la Bible à l'organisation de cellules d'appui à la lutte armée. Quelques unes ont entraîné toute leur famille dans l'aventure, après les protestations initiales d'un mari jaloux ou de parents violemment opposés à ce que leur fille côtoie autant d'hommes. D'autres ont été précipitées dans la clandestinité par un membre de leur 
famille poursuivi par les «escadrons de la mort». Mais pour la plupart, la guerre commence vraiment le jour où l'armée arrive et met le feu au village. Une partie des familles fuit vers la ville, l'autre vers la montagne, où la guérilla les accueille. Bien des femmes passent plusieurs mois aux côtés de la guérilla et poursuivies par l'armée, sans avoir véritablement choisi quoi que ce soit. Mais comme généralement d'autres membres de la famille sont du côté des compas guérilleros, elles donnent volontiers un coup de main - la force de l'habitude : nourrir ces jeunes gens, laver leurs vêtements, voire pour les plus jeunes et les plus dégourdies, prendre en charge les communications radios, apprendre quelques rudiments d'infirmerie... et pour les plus exceptionnelles, non sans difficultés, prendre un fusil pour venger leurs morts et pour lutter pour un meilleur futur pour les enfants du Salvador. Après 1982-1983, seules restent dans la guérilla proprement dite les plus jeunes et les plus déterminées. Les autres sont envoyées dans des camps de refugié-e-s dans les pays voisins ou près des villes, où le FMLN les organise en une solide base d'appui qui confectionne secrètement des sacs à dos, des uniformes et des explosifs, s'occupe des enfants des combattant-e-s, des orphelin-e-s et enfante les futur-e-s révolutionnaires.

5 A la fin de la guerre, en 1992, les 3.285 femmes démobilisées du FMLN ont entre 15 et 29 ans. $80 \%$ d'entre elles ont à leur charge des enfants de moins de douze ans, $29 \%$ sont chefs de foyer. Pendant la guerre, 29 \% d'entre elles étaient cuisinières ${ }^{4}$. Beaucoup d'autres étaient responsables des communications, infirmières ou chargées de l'éducation et de la propagande. Une infime minorité était combattante en première ligne, moins nombreuses encore étaient les gradées qui emmenaient leurs compagnons au combat. Les principales dirigeantes politico-militaires se comptent sur les doigts de la main: quatre commandantes qui deviennent députées en 1994, Rebeca Palacios, Nidia Díaz, Gina Cordón et Ana Guadalupe Martínez; plusieurs femmes tombées au combat comme Arlén Siú Guazapa, Suzana ou Ileana - une jeune paysanne de vingt ans qui dirigea l'éphémère bataillon exclusivement féminin «Silvia » ${ }^{5}$. Ces femmes ont joué un rôle d'exemple déterminant et ont puissament lutté pour favoriser la participation des femmes, mais leur éclat ne doit pas faire oublier la multitude des cuisinières et des agentes de liaison anonymes pour lesquelles la guerre révolutionnaire n'a été qu'un prolongement de leur rôle traditionnel de dévouement familial-maternel.

Douleur, dévouement et rébellion. Les fières ex-combattantes, les réfugiées et les veuves : des itinéraires de vie bouleversés

7 Le bilan de la guerre pour les femmes est extrêmement nuancé, selon leur origine sociale et géographique et leur degré d'engagement dans le conflit. D'un côté, on trouve une immense majorité des femmes qui ont subi la guerre sans la vouloir: la guerre a dévasté presque tout le nord-est du pays, théâtre principal des opérations militaires, et touché la quasi-totalité du territoire à un moment ou un autre. Les chiffres les plus fiables font état de près d'un million de personnes déplacées, réfugiées ou exilées. Les trois quarts étaient des femmes et leurs enfants, entassé-e-s dans des camps au Honduras ou près de San Salvador, ou égréné-e-s sans aucune aide dans les bidonvilles de la capitale et des pays voisins, voire jusqu'au Mexique, aux Etats-unis et au Canada. Pour ces femmes, dont presque aucune n'était sortie de son village avant la guerre, ces déplacements forcés ont représenté, plus qu'une opportunité de vie nouvelle, un crèvecœur et des souffrances infinies - sans parler de la rupture de l'unité familiale, leur unique référence jusqu'alors, et de la nécessité de trouver du travail dans un contexte difficile. Beaucoup d'entre elles ont su se montrer fortes et dignes, s'organisant 
remarquablement dans l'adversité, développant des modes de vie plus communautaires et des capacités que personne ne leur aurait reconnues en temps normal, apprenant à lire, à cultiver la terre ou à diriger la communauté ${ }^{6}$. Mais c'est aussi sur elles qu'est retombé la plus grande part du poids de la souffrance, du deuil, des angoisses quotidiennes. La répression s'est acharnée sur les plus engagées: des milliers de femmes ont été violées, torturées avec un luxe de violence, éventrées, assassinées ${ }^{7}$. L'ensemble de la population civile - dont les femmes constituent l'immense majorité- loin d'être épargnée, a été la véritable cible de la « guerre de basse intensité » : terreur, militarisation générale du pays, des esprits et de toutes les relations humaines, envahissement de la vie quotidienne par la violence. Les femmes voient leurs proches recrutés de force, assassiné-e-s, enlevé-e-s. La crise économique qui s'aggrave achève de limiter l'horizon à la simple survie.

Pour celles qui ont choisi de s'engager délibérément dans la lutte aux côtés du FMLN, le bilan est plus positif. Plusieurs dizaines des milliers d'entre elles restées dans les "territoires libérés » ou les campements de réfugié-e-s du FMLN ont expérimenté une vie nouvelle, plus collective et plus égalitaire, où leur participation sociale et politique a été encouragée - certes en bonne partie pour soutenir l'effort de guerre. Pour les militantes qui ont fait directement partie du FMLN, des avancées importantes ont eu lieu: alphabétisation, formations diverses, un certain partage de tâches avec les hommes, reconnaissance de capacités nouvelles de parole, de participation, voire de commandement, possibilité de choisir plus librement leurs compagnons de vie et surtout de contrôler davantage leur fécondité. Prenant courageusement à rebroussepoil la très forte moralité chrétienne paysanne, le FMLN permettait - ou incitait vivement à - la contraception et même l'interruption de grossesse chez ses " hommes " - ses combattants de sexe féminin. Parfois à leur corps défendant, mais souvent avec un certain soulagement, beaucoup de jeunes paysannes ont pu rompre avec leur destin séculaire de "se remplir d'enfants". L'immense majorité d'entre elles a continué à vouloir être mères, mais plus tard et d'une famille moins nombreuse, décidée avec le compagnon. Enfin, notamment à l'initiative d'un certain nombre de dirigeantes, le FMLN a lutté parmi ses troupes et ses bases contre le viol et même parfois contre la violence conjugale: pour les populations paysannes, dénoncer ces faits représentait déjà une révolution.

Cependant, la division sexuelle du travail s'est maintenue avec force : aux femmes l'arrière, la logistique, l'approvisionnement, l'alimentation, le soin aux blessé-e-s et le deuil, aux hommes le combat, la stratégie, la direction, la camaraderie virile, l'héroïsme et, pour certains hommes en position de pouvoir et de prestige, la polygamie facile. En dépit de la rhétorique révolutionnaire et de tentatives sincères et volontaristes de certain-e-s dirigeant-e-s, le FMLN a au moins autant reproduit que combattu les situations sociales pré-existantes. Sauf quelques heureuses exceptions, les hommes ont continué à commander aux femmes et les citadins scolarisés ont continué à diriger les bases paysannes. Les pesanteurs sociales sont extrêmement difficiles à balayer et la situation de guerre totale particulièrement cruelle a rendu considérablement plus difficile de lutter simultanément contre l'ennemi extérieur et contre le traditionalisme interne: si la participation à la lutte a permis à une certain nombre de femmes et d'hommes une mobilité sociale exceptionnelle, dans l'ensemble ce sont les personnes déjà les plus favorisées qui ont pu tirer le meilleur parti des brèches qui se sont ouvertes dans les destinées sociales traditionnelles ${ }^{8}$. 
10 Le prix à payer a été élevé : de nombreuses ex-combattantes, surtout celles qui ont le plus dérogé aux règles habituelles de comportement féminin, en devenant dirigeantes, en reléguant leurs projets de couple, de famille et de maternité à des jours meilleurs, se sont trouvées une fois la guerre finie devant d'énormes contradictions ${ }^{9}$. Alors que pour beaucoup d'hommes, finalement, la participation à la guerre s'inscrivait dans la continuité de leur socialisation, pour les femmes, elle a signifié des conflits et des ruptures considérables. Pendant la lutte, elles jouissaient d'un appui collectif matériel et d'une licence morale tacite pour laisser leurs enfants à l'arrière ou pour transgresser les codes amoureux ou familiaux "normaux », pour repousser leurs culpabilités et le regard critique de la société. Mais lorsque la guerre s'achève, ce sont elles qui doivent récupérer leurs enfants vieillis de douze ans et pleins d'amertume d'avoir été " abandonné-e-s », recoller les morceaux de famille qu'elles ont fait passer par les pires affres de l'angoisse quand elles étaient en prison, torturées ou parties quelque part dans les montagnes. Les hommes, les disparu-e-s, peuvent être considéré-e-s comme des héros révolutionnaires, mais la plupart des femmes ne sont que des êtres hybrides, étranges, qui ont sacrifié leur foyer et leur famille pour une promesse de futur qui n'a pas été tenue: il n'y a pas de victoire à mettre dans la balance. Le retour à la « normale » est d'autant plus difficile que la fin de la guerre, en 1992, les oblige à une reconversion rapide, un retour à la vie civile et à la recherche individuelle d'un emploi rémunéré, pour laquelle les hommes sont mieux équipés qu'elles, dans un contexte de crise économique profonde. Alors que le programme d'attribution de terres aux excombattant-e-s concerne surtout les hommes, qui peuvent également devenir policiers, chauffeurs, gardes du corps ou briguer des mandats politiques, les anciennes cuisinières et chargées des communications-radio du FMLN n'ont guère de qualifications à faire valoir, alors justement qu'elles retrouvent la responsabilité économique des enfants, et parfois d'autres parent-e-s. Elles-mêmes analysent avec incrédulité, douleur et révolte, a posteriori, les transformations que la lutte leur a imposées et que beaucoup n'ont jamais pleinement assumées: devenir dures, insensibles, non-maternelles, de vrais soldats-révolutionnaires-hommes ${ }^{10}$. Elles ne regrettent rien, mais elles souhaitent au moins faire fructifier cette expérience accumulée pour réorienter leur vie.

11 Pour qui travaillent les organisations de femmes ?

12 Des groupes de femmes existaient avant la guerre : en 1945, la Tribune féministe, le journal de l'Association de femmes démocratique du Salvador, revendique déjà le droit de vote des femmes - acquis en 1951. Entre 1957 et 1969, dans l'orbite du parti communiste salvadorien, la Fraternité des femmes lutte pour une amélioration de la condition féminine. De 1979 jusqu'au milieu des années quatre-vingts, l'Association de femmes du Salvador (AMES), liée à la principale organisation guérillère, se revendique du féminisme révolutionnaire et promeut activement la participation des femmes à la lutte - révolutionnaire bien plus que féministe : dans le cas d'AMES comme dans celui des autres organisations féminines, plus la guerre s'approfondit, plus les revendications propres sont mises de côté pour après la révolution et plus la participation des femmes est orientée vers l'effort de guerre ${ }^{11}$. Les multiples et souvent éphémères organisations féminines qui apparaissent jusqu'en 1989 sont toutes créées dans le sillage direct des organisations politico-militaires du FMLN ou par le gouvernement, pour obtenir de l'argent, des recrues et un poids moral plus grand dans le cadre d'un affrontement bipolaire gouvernement / guérilla qui laissait de côté toutes les autres contradictions 
sociales "secondaires». Les tentatives de certaines femmes pour lutter pour des revendications propres sont alors considérées comme divisionnistes, petitesbourgeoises ou influencées par des idées étrangères sans rapport avec les réalités et les nécessités des femmes salvadoriennes. Le FMLN permet le développement de la participation sociale, politique et même militaire des femmes, il la promeut souvent avec pragmatisme, mais en maintenant en grande partie la division sexuelle du travail révolutionnaire et en subordonnant les luttes des femmes à la lutte populaire.

Créée en 1986, la CONAMUS, qui lutte notamment contre la violence faite aux femmes, est la première depuis le début de la guerre à se réclamer à nouveau du féminisme. Après deux ans de tolérance et après que la CONAMUS a eu l'impudence de publier un document - pourtant interne - sur les droits sexuels des femmes et d'affirmer une certaine indépendance sur le plan financier et décisionnel, le parti dont elle dépend la fait rentrer dans le rang. Ce n'est qu'après l'offensive générale de 1989, «match nul » qui montre l'impossibilité d'une victoire militaire et l'épuisement de la population, que les protagonistes se convainquent que seule une solution politique est possible. Les négociations aboutissent à la paix début 1992. C'est dans ce contexte qu'à partir de 1990, de nouveaux groupes comme le Centre d'études féministes (CEF) et les Femmes pour la dignité et la vie (Dignas) et CeMujer, commencent aussi à se revendiquer du féminisme. Mais tant que dure la guerre, le féminisme demeure satanisé, tabou, pour la plupart des femmes et des groupes.

Début 1992, presque toutes les organisations de femmes sont directement issues de la lutte révolutionnaire, proche du FMLN. En lisant les Accords de paix, qui ne soufflent mot des femmes, beaucoup sont estomaquées. Les organisations les plus indépendantes, regroupées dans la Concertation de femmes pour la paix, l'égalité et la dignité, publient un communiqué de protestation dans lequel elles renvoient dos à dos le gouvernement et le FMLN.

Le modèle politique révolutionnaire s'est considérablement épuisé : la démocratisation interne du FMLN révèle certains fonctionnements patriarcaux ou corrompus qui suscitent la colère des militantes. La guerre cessant d'être l'unique préoccupation du pays, la réouverture matérielle et intellectuelle du pays permet aux questionnements que posent la chute du mur de Berlin et la décomposition de l'ancien bloc socialiste d'atteindre finalement le Salvador. La démocratisation - toute relative - de l'ensemble du pays et la dépolarisation politique créent un espace nouveau pour l'existence et l'expression d'une "société civile». Les organisations de femmes en deviennent rapidement la partie la plus active, à mesure qu'elles concquièrent leur autonomie financière, organisationnelle et surtout idéologique des partis dont elles étaient issues.

16 La VIe Rencontre féministe latino-américaine et des Caraïbes qui se tient au Salvador en novembre 1993 accélère aussi la marche au féminisme. Les groupes féministes d'Amérique centrale qui la préparent ensemble depuis 1991 l'inscrivent sciemment dans un processus de construction du mouvement régional et national. Elles réalisent ainsi une série d'activités préalables: création dans chaque pays d'un référent féministe (au Salvador, la Concertation de femmes pour la paix, l'égalité et la dignité), formations diverses, tenue d'une première rencontre nationale dans chaque pays puis d'une rencontre centro-américaine qui rassemble cinq cents femmes au Nicaragua en mars $1992^{12}$. Au Savador, la Concertation permet aux organisations les plus autonomes et aux féministes indépendantes de rompre leur isolement et de mettre en commun leurs expériences. Elles découvrent à un rythme accéléré et avec vertige les réflexions 
et les pratiques féministes du reste du continent, grâce aux multiples ateliers de discussion, souvent réalisés avec l'appui d'invitées féministes solidaires de toute la région. A l'été 1992, la Concertation décide de se revendiquer publiquement du féminisme et se lance dans la construction du « féminisme à la salvadorienne ».

La Concertation ne se cantonne pas à la préparation de cette VIe rencontre féministe continentale : dès 1993, elle est à l'origine de Mujeres 94, qui réunit plus de quarante groupes - la quasi totalité du mouvement des femmes du pays - pour élaborer une plateforme des femmes pour les élections générales de mars $94^{13}$. Cette plateforme inclut pour la première fois l'interruption volontaire de grossesse, la garantie constitutionnelle du respect des différentes options sexuelles et des quotas de $50 \%$ de femmes à tous les postes de responsabilités : trois points qu'aucun des partis, même le FMLN, n'acceptera d'inscrire dans son programme ${ }^{14}$. Toujours est-il qu'en moins de deux ans, les organisations de femmes se sont dotées d'une plateforme autonome, féministe, autour de laquelle elles ont su construire l'unité des organisations et des femmes les plus diverses du pays.

L'analyse de ce mouvement de femmes mobilisées désormais pour leur propre cause révèle deux phénomènes: sa composition multi-classiste et le poids des anciennes militantes révolutionnaires - certaines sorties des partis, d'autres demeurées fidèles. Il s'agit d'un mouvement massif, qui mêle des universitaires, des avocates, des femmes de classe moyenne urbaine, des femmes des secteurs populaires, des femmes des bidonvilles et des paysannes. Beaucoup de groupes continuent d'ailleurs à être organisés sur le modèle politique du FMLN et sur ses bases, par " secteurs ", à l'échelle nationale, avec des « groupes de bases » et des bureaux en ville - et la plupart tendent à se transformer en ONG. La volonté de construire et d'organiser, la recherche de l'unité, le goût pour le caractère massif et représentatif, l'intérêt pour la sphère politique et le pouvoir - un «autre pouvoir»- sont d'autres héritages du marxisme-léninisme salvadorien que ces militantes ont repris à leur compte.

On peut donc penser que la participation des femmes à la guerre civile révolutionnaire est loin d'être étrangère à l'apparition du féminisme salvadorien - qu'elle avait pourtant également freinée. Entre ce qui a été semé et ce qui a été détruit pendant la guerre, ce n'est en tout état de cause que dans la paix que les femmes parviennent finalement à penser à leurs propres problèmes et à rêver à voix haute. Le desserrement de l'emprise idéologique et matérielle du modèle de guerre civile révolutionnaire strictement bipolaire constitue le cadre dans lequel a pu apparaître un véritable mouvement social de femmes, d'où un fort courant féministe a émergé - entrainant le reste du mouvement des femmes dans un processus d'autonomisation que résume le slogan de Mujeres 94 : « Femmes, décidons par nous-mêmes! ».

\section{BIBLIOGRAPHIE}

ALEGRÍA, Claribel ; FLAKOLL, D.J. 
1987 No me agarran viva, La mujer salvadoreña en la lucha, San Salvador, UCA.

ARAUJO, Ana María.

1980 Tupamaras, Des femmes de l'Uruguay, Paris, Des femmes.

CAÑAS, Mercedes.

Printemps 1992 En El Salvador, muchas mujeres : gracias a la guerra, salimos de las cocinas, dónde sólo estábamos quemándonos, México, Uno Más Uno et ronéo.

CEMUJER.

1992 Norma, vida insurgente y feminista, San Salvador, Edition CeMujer.

DÍAZ, Nidia, Commandante.

1988 Nunca estuve sola, San Salvador, UCA Editores.

DIVERS.

1989 A dream compells us. Voices of salvadoran women, San Francisco, New America Press.

FALQUET, Jules-France.

1996 « Entre rupture et reproduction : femmes salvadoriennes dans la guerre révolutionnaire (1981-1992) », Paris, Nouvelles questions féministes; Vol. 17, n².

GARAÍZABAL, Cristina, VÁSQUEZ, Norma.

1994 El dolor invisible de la guerra. Una experiencia de grupos de auto-apoyo con mujeres salvadoreñas, Madrid, Talasa, Hablan las mujeres, Mujeres por la dignidad y la vida.

HARNECKER, Marta.

juillet 1993 Entrevista a la Comandante Rebeca, Retos de la mujer dirigente, La Habana, Colección Letra viva (20 p.).

JIMÉNEZ, Liliam.

septembre 1984 La mujer revolucionaria en El Salvador, México, Plural, n¹56.

MARTÍNEZ, Ana Guadalupe, Commandante.

$1981 \mathrm{El} \mathrm{Salvador,} \mathrm{une} \mathrm{femme} \mathrm{du} \mathrm{front} \mathrm{de} \mathrm{libération} \mathrm{témoigne} \mathrm{(traduction} \mathrm{française} \mathrm{de} \mathrm{Las} \mathrm{cárceles}$ clandestinas de El Salvador, San Salvador, 1980), Paris, Des Femmes.

MUJERES 94.

31 de agosto 1993 Plataforma de las mujeres salvadoreñas, San Salvador.

MUJERES POR LA DIGNIDAD Y LA VIDA.

1995 Las mujeres ante, con, contra, desde, sin, tras... el poder político, San Salvador, brochure réalisée avec l'appui d'Oxfam (GB) et Novib (Hollande).

MURGUIALDAY, Clara.

1990 Nicaragua, revolución y feminismo (1977-1989), Madrid, Editorial Revolución, Colección Hablan las mujeres.

NAVAS TURCIOS, María Candelaria.

février 1987 Las organizaciones de mujeres en El Salvador, 1975-1985, Mexico, Tesis para la Maestría en Estudios latinoamericanos, Facultades de Ciencias sociales y políticas, UNAM. 
NAVAS TURCIOS, María Candelaria; RIVERA, Ana Kelly; ORTIZ CAÑAS, Edy Arelí; DOMÍNGUEZ MAGAÑA, Liza.

1995 Valió la pena ?! Testimonios de salvadoreñas que vivieron la guerra, San Salvador, Editorial Sombrero Azul.

ROVÍRA, Guiomar.

1996 Mujeres de maís, Barcelona, Virus Editorial.

SORO, Julio; GONZALEZ, Lissette; OSORIO, Clara Luz; PORTILLO, Elvira; VEGA, Ana Dora de.

1993 Cómo los partídos politicos incluyen a las mujeres en sus plataformas políticas, San Salvador, Red por la unidad y el desarrollo de las mujeres salvadoreñas, Prodere El Salvador.

THOMSON, Marilyn.

1986 Women of El salvador, the price of freedom, London, USA, Zed Books Ltd, publié avec le concours de la Comisión de Derechos Humanos de El Salvador (non-gouvernementale) et de War on want.

VÁSQUEZ, Norma; MURGUIALDAY, Clara.

avril 1992 Encuentro centro-americano de mujeres : más que un encuentro, un proceso de construcción del feminismo centroamericano, ronéo, $14 \mathrm{p}$.

\section{NOTES}

1. En Amérique du Sud, les guérillas sont plutôt urbaines. Des femmes de classe moyenne y ont une participation importante mais relativement subordonnée, comme le donne à penser par exemple le témoignage des femmes Tupamaras : Araujo, 1980. L'exception serait le Sendero luminoso du Pérou, où selon la revue Fempress, les femmes constituent $40 \%$ des effectifs de la guérilla et sont particulièrement actives au sein des tristes « commandos de aniquilamiento ». Pour le Guatemala, peu de témoignages écrits de femmes, pour le Nicaragua : Murguialday, 1990 et pour le Chiapas, Mexique : Rovíra, 1996. L'EZLN compte environ $30 \%$ de femmes indiennes dans ses troupes.

2. Une des premières Salvadoriennes à relater cette lutte est Liliam Jiménez : Jiménez, 1984. Elle était également poétesse et auteure du premier livre jamais écrit sur la situation des femmes au Salvador, La condición de la mujer salvadoreña. Publié en 1962 au Mexique, le livre fait immédiatement l'objet d'un embargo au Salvador.

3. Hommages posthumes à Eugenia et à Norma Guirola et témoignages de la vie clandestine des militantes urbaines de cette période : Alegría ; Flakoll, 1987. CeMujer, 1992.

4. Selon l'ONUSAL, cité par Mujeres por la dignidad y la vida, 1995.

5. On trouve une interview d'elle et de beaucoup d'autres dans Divers, 1989. Ileana a été tuée en combat au printemps 1984, à l'âge de 21 ans. On verra aussi Thomson, 1986.

6. Cañas, 1992.

7. Martínez, 1981. Díaz, 1988.

8. Falquet, 1996.

9. Sur les difficultés et la vie d'une femmme dirigeante politico-militaire, lire témoignage piquant de Rebeca Palacios : Harnecker, 1993.

10. Garaízabal ; Vásquez, 1994.

11. Navas, 1987.

12. Vásquez; Murguialday, 1992. 
13. Mujeres 94, 1993.

14. Soro, Julio ; Gonzalez, Lissette ; Osorio, Clara Luz ; Portillo, Elvira ; Vega, Ana Dora

de, 1993.

\section{RÉSUMÉS}

La guerre civile révolutionnaire au Salvador a fait irruption dans la vie des femmes et les a précipitées hors des cuisines. Si un certain nombre d'entre elles en ont profité pour bousculer leur destin séculaire, tenter de prendre en mains leur propre vie et l'avenir du pays en participant activement au projet révolutionnaire, beaucoup d'autres se sont contentées de subir, non sans héroïsme, la violence, la terreur et la misère supplémentaire produites par la guerre. La guerre n'a rien laissé intact, mais les rapports sociaux de sexes, particulièrement résistants, n'ont pas été modifiés radicalement. Ce n'est qu'avec le retour à la paix que, fortes de leur expérience acquise dans la lutte, des femmes ont véritablement pu commencer à lutter pour leurs propres besoins et rêves et à construire un fort mouvement de femmes qui s'oriente vers des positions féministes autonomes.

The salvadorian revolutionnary civil war irrupted into women's lives and threw them out of the kitchens. Some of them took advantage of the situation to try to modificate their traditional destiny and take in their own hands their lives as well as the whole country's life, through active participation to the revolutionary project. But the majority of women only suffered in quite a undesired, even if heroical way, the violence, terror and additional misery produced by the war. The war caused very deep transformations in every aspect of personal, economical, social and political life, but the gender system, which is especially hard to tranform, didn't change that much. It is only in the peace time that women, strengthened by the experience they acquired in the struggle, have been able to start claiming for their own needs and dreams and to build a strong women's movement, heading towards autonomous feminist positions.

\section{AUTEUR}

\section{JULES FALQUET}

Jules FALQUET prépare un doctorat de sociologie sur le mouvement des femmes et sa composante féministe au Salvador, où elle a vécu entre 1992 et 1994. Elle s'est intéressée plus particulièrement à la prise en compte des femmes dans les programmes électoraux des partis salvadoriens, aux politiques de population et aux droits sexuels et reproductifs des salvadoriennes. En 1990, elle avait travaillé sur les rapports entre la scolarisation des femmes indiennes et la résistance culturelle des populations indiennes dans le Chiapas, au Mexique. Elle suit actuellement avec intérêt la participation des femmes dans la lutte zapatiste. 